\title{
A dp53/JNK-dependant feedback amplification loop is essential for the apoptotic response to stress in Drosophila
}

\author{
E Shlevkov ${ }^{1}$ and G Morata*,
}

Programmed cell death (apoptosis) is a conserved process aimed to eliminate unwanted cells. The key molecules are a group of proteases called caspases that cleave vital proteins, which leads to the death of cells. In Drosophila, the apoptotic pathway is usually represented as a cascade of events in which an initial stimulus activates one or more of the proapoptotic genes (hid, rpr, grim), which in turn activate caspases. In stress-induced apoptosis, the dp53 (Drosophila p53) gene and the Jun N-terminal kinase (JNK) pathway function upstream in the activation of the proapoptotic genes. Here we demonstrate that $d p 53$ and JNK also function downstream of proapoptotic genes and the initiator caspase Dronc (Drosophila NEDD2-like caspase) and that they establish a feedback loop that amplifies the initial apoptotic stimulus. This loop plays a critical role in the apoptotic response because in its absence there is a dramatic decrease in the amount of cell death after a pulse of the proapoptotic proteins Hid and Rpr. Thus, our results indicate that stress-induced apoptosis in Drosophila is dependant on an amplification loop mediated by dp53 and JNK. Furthermore, they also demonstrate a mechanism of mutual activation of proapoptotic genes.

Cell Death and Differentiation (2012) 19, 451-460; doi:10.1038/cdd.2011.113; published online 2 September 2011

Apoptosis is a process of major physiological importance, conserved in the animal kingdom, aimed to eliminate cells. It performs developmental roles, reported in insects and vertebrates, and also functions as a safeguard mechanism to remove abnormal cells or cells damaged by stress events. ${ }^{1,2}$ The executive role in apoptosis is played by a group of cysteine proteases, termed caspases, which destroy many vital cell proteins, thus causing cell death. ${ }^{3}$ All animal cells contain them, but their activity is normally prevented by the inhibitor of apoptosis proteins (IAPs). ${ }^{4,5}$ Thus, a key regulatory step in apoptosis is the control of IAP function.

In Drosophila, the initiation of apoptosis is achieved by the activation of one or more of a group of proapoptotic genes reaper (rpr), head involution defective (hid) and grim (Figure 1a). Their products bind to Drosophila IAP-1 (Diap1) and interfere with its function, releasing the caspases. ${ }^{6}$ A target of Diap1 is the initiator caspase-9 ortholog Dronc (Drosophila NEDD2-like caspase). ${ }^{7}$ The only known Dronc substrates to date are effector caspases Dcp-1, Drice (Drosophila interleukin converting enzyme) and Diap1 itself. ${ }^{6}$

The $p 53$ gene is a major proapoptotic factor in both Drosophila and vertebrates. ${ }^{8}$ In mammalian cells, the P53 protein has other nonapoptotic functions; it is involved in antiproliferative responses, including cell-cycle arrest and senescence. $^{9,10}$ In contrast, the Drosophila homolog of $p 53$, called $d p 53$, has mostly a proapoptotic role, although some effect on cell-cycle regulation has been noticed in overexpression experiments. ${ }^{11}$ It has been reported that $d p 53$ activates the transcription of $r p r$ in response to radiationinduced DNA damage to initiate apoptosis, ${ }^{12}$ and that overexpression of $d p 53$ in the eye induces Hid-mediated apoptosis. ${ }^{11}$ Thus, $d p 53$ is currently depicted upstream of the apoptotic cascade (Figure 1a). Another major proapoptotic factor in Drosophila and vertebrates is the Jun N-terminal kinase (JNK) pathway. ${ }^{13}$ In Drosophila, this pathway plays a prominent role in inducing apoptosis and is activated in a variety of contexts, including tumor suppression. ${ }^{14,15}$

The scheme in Figure 1a accounts for most of the features of Drosophila apoptosis, but recent findings have suggested the existence of additional complexity. These originate from experiments in which effector caspase activity was blocked by the baculovirus protein P35. ${ }^{16}$ The presence of P35 does not change the apoptotic nature of these cells (called 'undead' cells), but they remain alive, and this is what facilitates the study of their properties.

A significant observation is that after a brief apoptotic stimulus, X-radiation or a 2 to $3 \mathrm{~h} 37^{\circ} \mathrm{C}$ heat shock, cells containing $\mathrm{P} 35$ remain in a prolonged apoptotic status: $72-96 \mathrm{~h}$ after the end of the stimulus, when normally cell death is already undetectable, they still show expression of the proapoptotic gene hid and active Drice. ${ }^{17}$ Furthermore, they show persistent expression of the signalling genes

\footnotetext{
${ }^{1}$ Centro de Biología Molecular, CSIC-UAM, Madrid, Spain

*Corresponding author: G Morata, Centro de Biología Molecular, CSIC-UAM, Nicolas Cabrera 1, Madrid 28049, Spain. Tel: + 3491 196 4696; Fax: + 3491 196 4420; E-mail: gmorata@cbm.uam.es

Keywords: apoptosis; p53; JNK; Dronc; Drosophila; imaginal discs

Abbreviations: Diap1, Drosophila inhibitor of apoptosis protein 1; dp53, Drosophila p53; dp53-i, dp53 RNA interference; dpp, decapenthaplegic; Drice, Drosophila interleukin converting enzyme; Dronc, Drosophila NEDD2-like caspase; dronc-i, dronc RNA-interference; en, engrailed; gfp, green fluorescent protein; hep, hemipterous; hid, head involution defective; hs-hid, heat shock-head involution defective; hs-rpr, heat shock-reaper, IAP, inhibitor of apoptosis protein; JNK, Jun N-terminal kinase; puc, puckered; ROI, region of interest; rpr, reaper, sal, spalt, TUNEL, TdT-mediated fluoroscein-dUTP nick end labelling; UAS, upstream activation sequence; $w g$, wingless

Received 16.2.11; revised 07.7.11; accepted 25.7.11; Edited by S Kumar; published online 02.9.11
} 
Hid

a
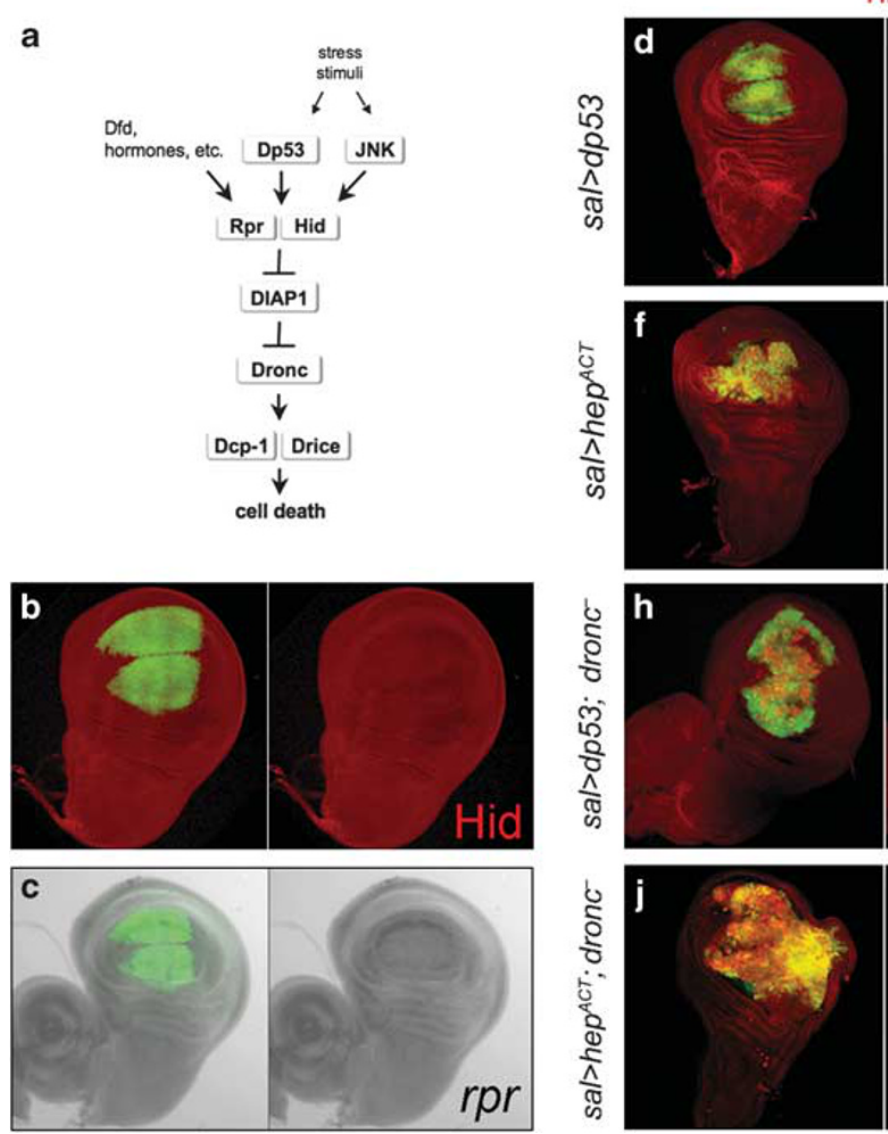
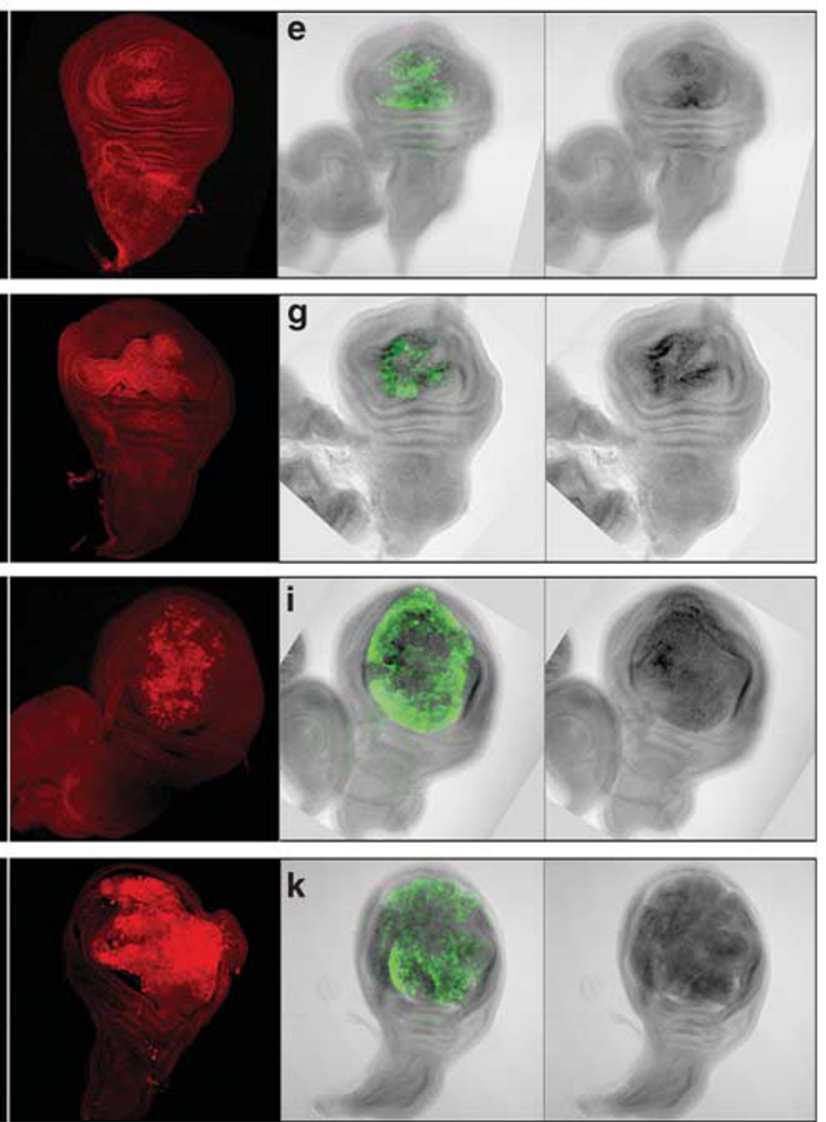

Figure 1 Proapoptotic genes are activated by Dp53 and the JNK pathway. (a) Simplified scheme of the apoptotic pathway in Drosophila. Upstream activators like Dp53 and the JNK pathway induce one or more of the proapoptotic genes (only hid and rpr are shown in the scheme but there are other related genes, grim and sickle). The inactivation of the apoptosis inhibitor Diap1 by the proapoptotic proteins allows the proteolytic activation of the Dronc, Dcp-1 and Drice caspases and subsequent cell death (see Hay and Guo ${ }^{6}$ for a more detailed description). (b and $\mathbf{c}$ ) Control discs of genotype spalt ${ }^{E P V}$-Gal4 $>$ UAS-GFP doubly stained for GFP and Hid and for GFP and $r p r$ transcripts. There is neither hid nor rpr activity in the saldomain, labelled green. (d-g) Activation of hid and rprby Dp53 and JNK pathway. (d and e) Discs of genotype spalt ${ }^{E P V}$ Gal4 > UAS-GFP UAS-dp53 showing Hid protein (d) and rpr transcription (e) in the sal domain. (f and $\mathbf{g}$ ) Disc of genotype spalt ${ }^{E P} V$-Gal4 $>$ UAS-GFP UAS-hep ${ }^{A C T}$ showing accumulation of the Hid protein (f) and rpr activity $(\mathbf{g})$ in the sal domain. (h-k) The activation of hid and rpr by Dp53 and JNK pathway in dronc mutant discs. (h) Disc of genotype spalt ${ }^{E P V}$-Gal4 > UAS-GFP UAS-dp53 dronc demonstrating the presence of the Hid protein in the expanded sal domain. (i) Disc of the same genotype as in (h) also showing rpr transcription in the expanded sal domain. (j and k) spalt ${ }^{E P V}$-Gal4 > UAS-GFP UAS-hep ${ }^{A C T}$ dronc $^{-}$discs also showing gain of Hid (j) and rpr (k) in the sal domain. The sal domain in the dronc mutant discs becomes enlarged because of the ectopic expression of $w g$ and dpp induced by JNK ${ }^{22}$

decapenthaplegic (dpp) and wingless $(w g),{ }^{18}$ another feature of apoptotic cells.

The persistence of the apoptotic program in undead cells suggests a maintenance mechanism. In this context, it is of interest to consider the function of $d p 53$ and the JNK pathway. dp53 becomes activated in response to irradiation and in turn directly activates the expression of $r p r .^{12}$ However, there is evidence that in undead cells $d p 53$ gene is activated by Dronc. ${ }^{19}$ The function of $d p 53$ both upstream and downstream Dronc suggested that it is part of a feedback loop aimed to maintain the apoptotic program. ${ }^{19} \mathrm{~A}$ similar argument can be made with the JNK pathway: irradiation induces JNK, which in turn induces $r p r,{ }^{20}$ but JNK also functions downstream Dronc to activate $d p p$ and $w g$ in undead cells. ${ }^{21,22}$ These findings place JNK function genetically both upstream and downstream Dronc.

Together, the preceding observations indicated the existence in undead cells of a feedback loop that involves $d p 53$ and JNK and that maintains the apoptotic program. However, the biological significance of this loop was unclear because the undead cells are aberrant apoptotic cells generated by artificial interference with effector caspase activity. We have performed experiments to test whether there is a similar feedback loop involving $d p 53$ and the JNK pathway in cells embarked in a normal apoptotic program and if so, which is its biological function. Our results show that a $d p 53 / \mathrm{JNK}$ mediated feedback loop indeed occurs in stress-induced apoptosis. Furthermore, they demonstrate that it is essential for the completion of the cell death program, for interfering with the loop results in a dramatic reduction of cell death after an apoptotic stimulus.

\section{Results}

A feedback loop in apoptotic cells. We have investigated the possible existence of a feedback loop by inducing apoptosis in cells in which the apoptotic program is not 
interfered with P35. We have made use of the Gal4/UAS method ${ }^{23}$ to drive expression of the different genes involved. As a routine we used the $s a l^{E P V}-G a l 4$ line, ${ }^{24}$ which directs expression only in part of the wing imaginal disc and acts relatively late in the development. It allows normal larval viability after apoptotic induction. Moreover, apoptotic cells can be visualized in third-instar discs, what facilitates the study of their behavior.

As $d p 53$ and the JNK pathway appear to be key factors in apoptosis, we first examined their ability to induce proapoptotic genes. As expected from previous work, ${ }^{11,12,20,25}$ both dp53 and JNK can activate hid and rpr. in sal> dp53 and in sal> hep ${ }^{A C T}$ discs (where hep ${ }^{A C T}$ provides constitutive activity of the JNK pathway ${ }^{26}$ ), the two proapoptotic genes become expressed in the spalt (sal) domain (Figures $1 \mathrm{~d}-\mathrm{g}$ ). The induction also occurs in discs of the null allelic combination dronc ${ }^{124} /$ dronc $^{129}$ (hereafter referred to as dronc $^{-}$), indicating that hid and rpr induction by $d p 53$ and JNK is not mediated by a feedback loop (Figures $1 \mathrm{~h}-\mathrm{k}$ ).

Having shown that $d p 53$ and JNK can activate hid and rpr, we then checked whether the latter might reciprocally induce dp53 and JNK. The results are illustrated in Figure 2: in sal > hid discs there is transcription of $d p 53$ and JNK activity in the sal domain (Figures 2c and d). However, and importantly, the activation of both $d p 53$ and JNK by Hid does not occur in dronc $^{-}$mutants (Figures $2 \mathrm{f}$ and $\mathrm{g}$ ) indicating that it is downstream of and requires dronc function. These results demonstrate that the activation of $d p 53$ and JNK downstream dronc is a component of the apoptotic program.

The results of the preceding experiments indicate that $d p 53$ and JNK can activate hid and rpr, and that hid can in turn induce dp53 and JNK through Dronc. It follows that both hid and rpr should be able to activate each other. This is indeed the case: forcing expression of hid in the sal domain gives rise to rpr activity (Figure 2e), and this activation requires dronc function (Figure $2 \mathrm{~h}$ ). This finding suggests that $d p 53$ and JNK pathway function downstream dronc to feedback the apoptotic program by increasing the levels of Hid and Rpr products. In addition, this feedback provides a mechanism of mutual activation among proapoptotic genes. Complementary experiments forcing $r p r$ expression yielded similar results, included in Supplementary Figure S1.

We finally tested the existence of interactions between dp53 and JNK. The experiments consisted of forcing

Figure 2 The proapoptotic factor Hid activates dp53, the JNK pathway and $r p r$ in a dronc-dependant manner. (a and $\mathbf{b}$ ) Control discs of genotype spalt ${ }^{E P v}$. Gal4 > UAS-GFP doubly stained for GFP and dp53 transcript (a) and for GFP and the phosphorylated form of JNK/Basket (b). (c and d) The effect of Hid on dp53 transcription and JNK pathway activity. (c) spalt ${ }^{E V}$-Gal4 > UAS-GFP UAS-hid disc showing dp53 expression, revealed by in situ hybridization, in cells expressing hid, labelled by anti-Hid in red. (d) In the spall ${ }^{E P V}$-Gal4 > UAS-GFP UAS-hid disc, there is also JNK activity in the sal domain in coexpression with Hid, as revealed with the antibody that recognizes the phosphorylated form of JNK/Basket (gray). The (e) row illustrates the activation of rpr by Hid. Following the same scheme as in the first two rows, forcing expression of hid in the sal domain results in rpr activation, revealed by in situ hybridization. (f-h) The result of forcing hid in dronc ${ }^{-}$discs is shown. (f) Disc of genotype spalt ${ }^{E P V}$-Gal4 > UAS-GFP UAS-hid dronc ${ }^{-}$showing Hid expression in the sal domain but no dp53transcription. The (g) row demonstrates that JNK activity is absent in the spalt ${ }^{P{ }^{P V}}$-Gal4 > UAS-GFP UAS-hid dronc ${ }^{-}$disc, and (h) that in dronc mutant discs $r p r$ remains silent
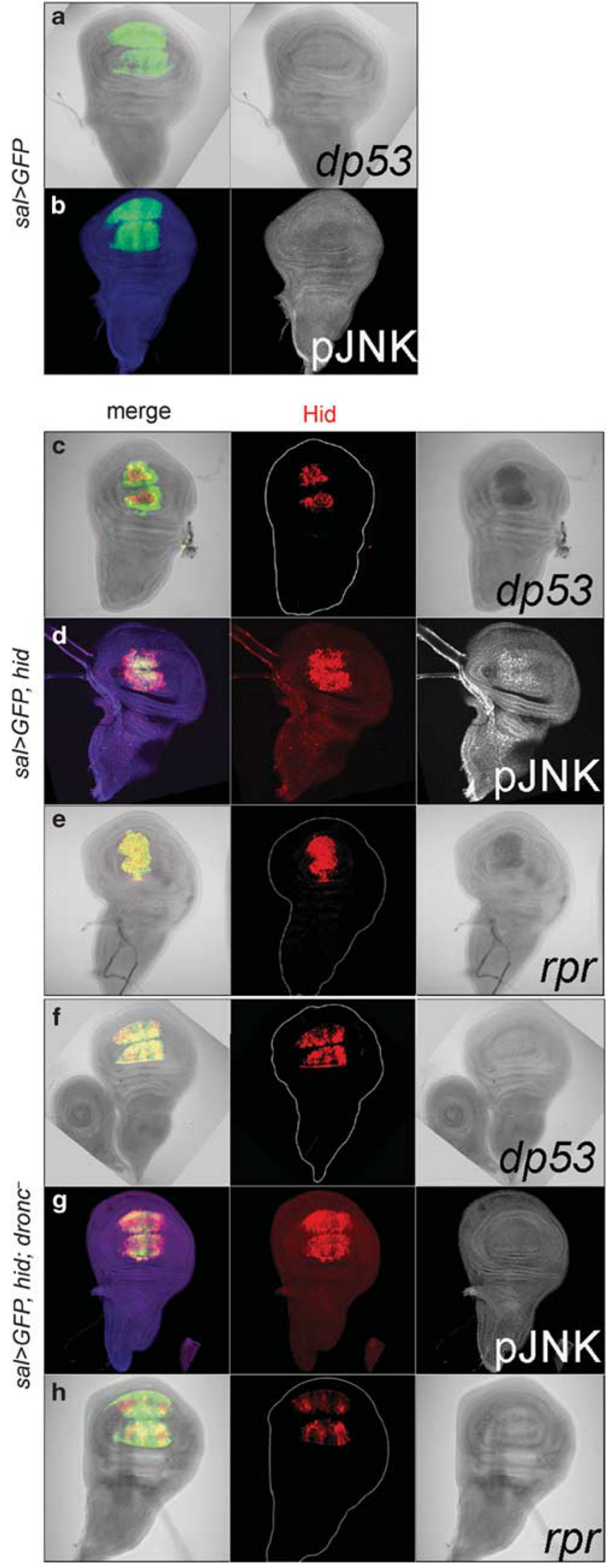

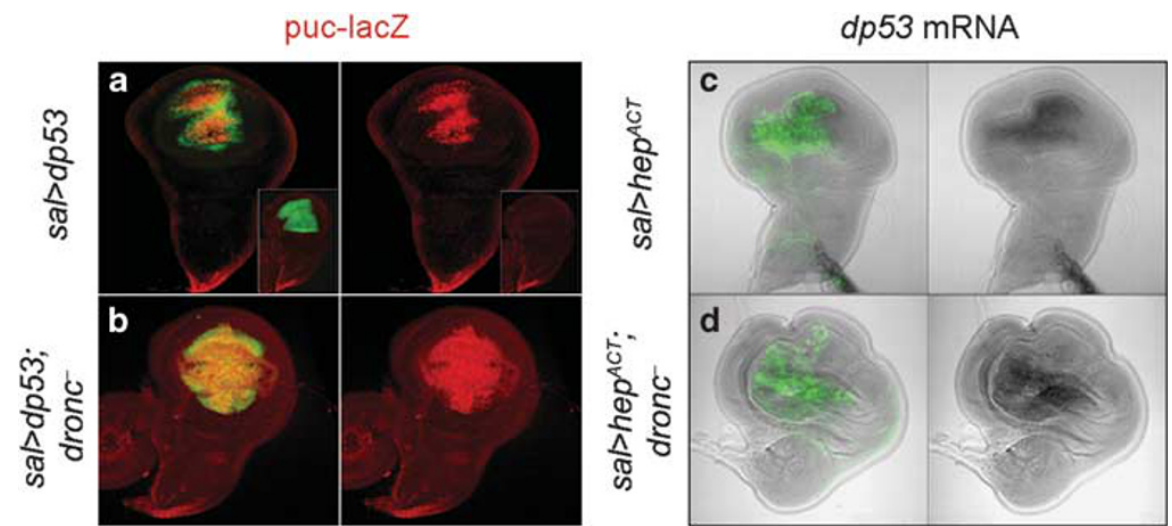

Figure $3 d p 53$ and JNK are able to activate each other independently of dronc function. (a and $\mathbf{b}$ ) In sal $>d p 53$ (spalt ${ }^{E P v}$-Gal4 $>$ UAS-dp53 UAS-GFP; puc-lacZ/ + ) and sal> dp53 dronc $^{-}$(spalt ${ }^{\text {Evv }}$-Gal4 > UAS-dp53 UAS-GFP; puc ${ }^{E 69}$-lacZ dronc ${ }^{124} /$ dronc $^{129}$ ) discs, the JNK pathway becomes activated, as revealed by the puc-LacZ marker. In otherwise wild-type discs, puc-lacZ expression is restricted to the most proximal cells (inset). Conversely, driving JNK expression in the sal domain activates $d p 53$ transcription in both rronc $^{+}$and dronc $^{-}$discs (c and $\left.\mathbf{d}\right)$

expression of either $d p 53$ or activating the JNK pathway in the sal domain and then checking JNK and dp53 activity, respectively. The experiments were performed in dronc $^{+}$ and dronc $c^{-}$mutant background to test whether their interactions were mediated by the loop. The unexpected result is illustrated in Figure 3 and shows that these two factors can activate each other even in dronc $^{-}$mutant discs.

The feedback loop is essential for the completion of the apoptotic program. Having demonstrated the existence of a feedback loop involving $d p 53$ and JNK in cells undergoing the normal apoptotic program, the next step was to test whether it has a functional role. The rationale of the experiments was to induce a brief pulse of proapoptotic genes such as hid or rpr and then to study the apoptotic response of the wing disc in the presence and absence of dronc, dp53 or JNK.

We have used two gene constructs bearing a minimal heat shock promoter directing expression of either hid or rprcoding regions. They allow forcing proapoptotic activity simply by a temperature shift (pulse of $30 \mathrm{~min}$ at $37^{\circ} \mathrm{C}$ ) and independently of upstream controls. The apoptotic response was measured by examining Drice protein levels and TUNEL (TdT-mediated fluoroscein-dUTP nick end labeling) staining $4 \mathrm{~h}$ after the end of the heat pulse, and also the accumulation of the Hid protein.

The experiments were performed in parallel with the two heat shock constructs. They involved the generation of the following genotypes: (1) en>gfp; hs-hid (control), (2) en> dronc-i; hs-hid, (3) en > dp53-i; hs-hid and (4) en > puc; hs-hid and also the same series with the heat shock-reaper (hs-rpr) construct. The usage of the en-Gal4 driver in these experiments permits to interfere with the loop in the posterior compartments, leaving the anterior ones as controls. In genotypes 2 and 3, we interfered with transcription of dronc or dp53 using RNA-i (see Materials and Methods). In the case of $d p 53$ we observed that it has the same effect as the dominant negative form (Supplementary Figure S2), thus confirming its specificity. In genotype 4 , the overexpression of the negative regulator puckered (puc) causes the strong reduction of JNK activity. ${ }^{27}$ As the results obtained in the two series of experiments are very similar, we only describe in detail the heat shock-head involution defective (hs-hid) series (Figures 4 and 5). The results of the hs-rpr experiments are shown in Supplementary Figure S3.

In the control genotype en>gfp; hs-hid, the heat shock pulse generates a strong apoptotic response, indicated by the high levels of co-extensive anti-Caspase-3 and TUNEL staining in the entire disc (Figures $4 a$ and $a^{\prime}$ ). Expectedly, in en $>$ dronc-i; $h$ s-hid discs, Caspase-3 and TUNEL levels in the posterior compartment after heat shock are low (Figure 4b), because apoptosis is blocked by the lack of dronc activity.

The significant result is that in en>dp53-i; hs-hid and en > puc; hs-hid discs, there is a very clear reduction of the amount of cell death in the posterior compartment in comparison with the control anterior compartments (Figures $4 \mathrm{c}$ and $\mathrm{d}$ ). This finding suggested that most of the cell death caused by hid is mediated by the amplification loop established by $d p 53$ and JNK. It also suggested that the loop might feed back to the endogenous hid gene. Therefore, we examined Hid protein levels in the same genotypes of above. These results are shown in Figure 5. Whereas in en $>$ gfp; hs-hid discs the heat shock generates high levels of Hid protein (Figures $5 \mathrm{a}$ and e), in en $>$ dronc-i; hs-hid Hid is almost undetectable in the posterior compartment (Figure $5 \mathrm{~b}$ and e). Similarly, there is a clear reduction of Hid protein in the posterior compartments of en>dp53-i; hs-hid and en $>$ puc; hs-hid discs (Figures $5 \mathrm{c}-\mathrm{e}$ ), suggesting that the accumulation of Hid protein after heat shock is mediated by the dronc/dp53/ JNK loop. The loop-mediated accumulation in Hid protein can also be detected in the $h s-r p r$ experimental series: in en $>d$ ronc-i; hs-rpr and en>puc; hs-rpr discs, there is a drastic reduction of Hid protein in the posterior compartments in comparison with the anterior ones (Supplementary Figures $\mathrm{S} 3 \mathrm{~b}$ and $\mathrm{c}$ ). We also examined the apoptotic response in haltere and leg discs, seeking to confirm the results in the wing disc. As shown in Supplementary Figure S4, we obtain similar results.

The preceding experiments strongly suggest that the proapoptotic proteins are able to induce their own genes through the loop. However, there was the possibility that the 


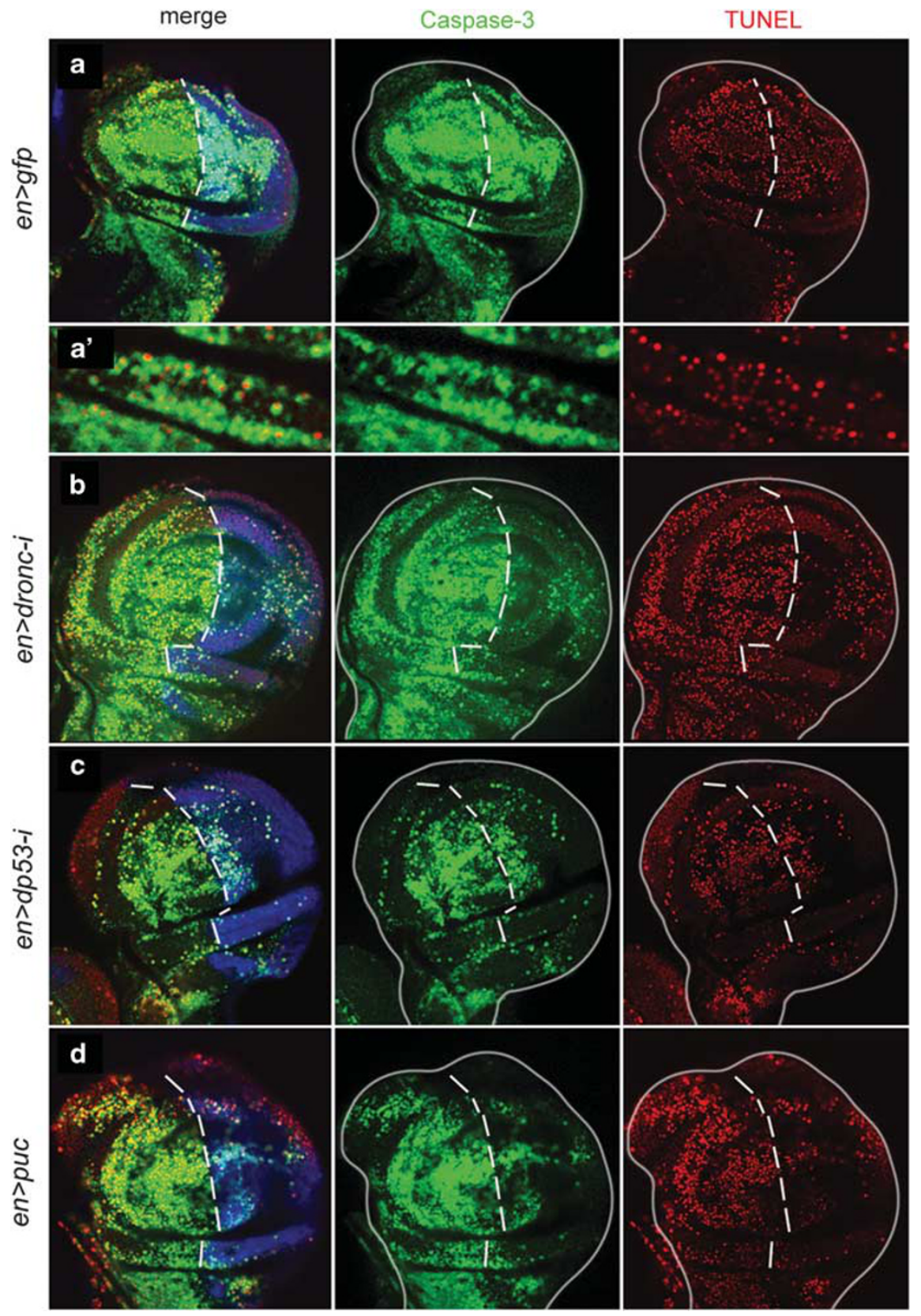

Figure 4 Apoptotic response to a brief pulse of hid activity. (a) en-Gal4 > UAS-GFP; hs-hid wing disc showing high levels of anti-Caspase-3 (green) and TUNEL (red). GFP staining is shown in blue. A magnification is shown in $\left(\mathbf{a}^{\prime}\right)$ to appreciate the concordance between anti-Caspase-3 and TUNEL staining. (b) en-Gal4 $>$ UAS-GFP UAS-dronc RNA-i; hs-hid showing a much reduced response in the posterior compartment. In the discs of genotype en-Gal4> UAS-GFP UAS-dp53 RNA-i; hs-hid (c) or en-Gal4 > UAS-GFP UAS-puc; hs-hid (d), the apoptotic response in the posterior compartments is also much reduced. Note that TUNEL and anti-Caspase-3 staining are highly concordant in all cases

low levels of Hid protein in the posterior compartments, in which dronc/dp53/JNK are inhibited, are because of either increased degradation of the exogenous Hid protein or decreased translation of the exogenous hid transcript. We have tested these possibilities by introducing the P-element insert id $^{05014}$-lac $Z^{28}$ in the $h s$-hid experiment and examining the lac $Z$ activity in anterior and posterior compartments after heat shock. The higher lac $Z$ expression levels in the anterior compartment clearly indicate transcriptional upregulation of the endogenous gene (Figure 6a). Furthermore, we have tested the response to $h s$-hid of the hid $^{20-10}$-lacZ, a construct that can be activated by Dp53. ${ }^{11}$ As shown in Figure 6c, lacZ expression is induced by the heat shock, supporting the idea that Dp53 is involved in the activation of the endogenous hid gene after a pulse of hid. Moreover, we find that the Hid protein is present at high levels immediately after the end of the heat shock and that those levels are similar in the anterior and posterior compartments of en $>$ dronc-i, hs-hid discs (Supplementary Figure S5). 


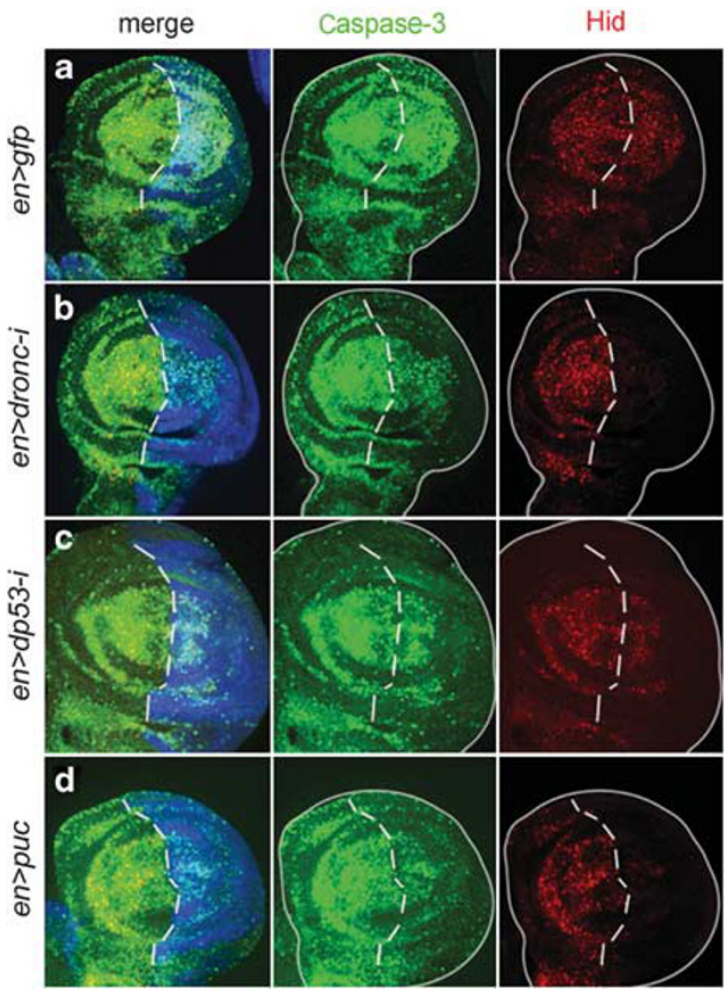

e
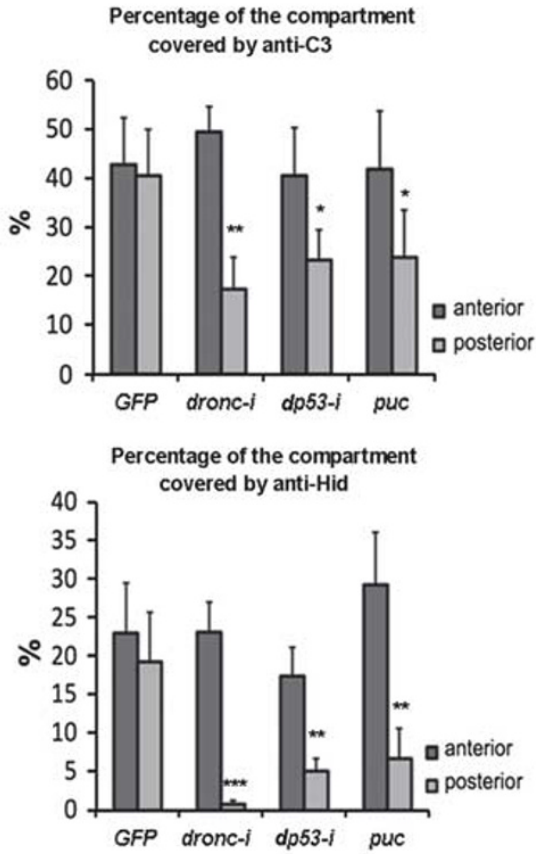

Figure 5 Loop-mediated amplification of Hid levels after a pulse of hid. (a) Control en-Gal4> UAS-GFP; $h s$-hid flies. The expression of GFP is shown in blue. The 30 min heat shock induces high levels of Caspase-3 (green) and Hid (red), both in the anterior and posterior compartments. (b) en-Gal4> UAS-GFP UAS-dronc RNA-i; hs-hid disc showing a large reduction of Caspase-3 and Hid activity in the posterior compartment because of the suppression of dronc function. The third panel clearly indicates that most of the Hid protein visible after the heat shock is generated by the feedback loop. (c) A en-Gal4 > UAS-GFP UAS-dp53 RNA-i; hs-hid disc showing reduction of Caspase-3 and Hid in the posterior compartment, where dp53 function is diminished. (d) en-Gal4 > UAS-GFP UAS-puc; hs-hid disc with reduced levels of Caspase-3 and Hid in the posterior compartment. (e) The results of quantitative measurements of Caspase-3 and Hid activities in the genotypes studied. The percentage of the area of each compartment covered by the staining with anti-Caspase-3 or anti-Hid was calculated as indicated in the Materials and Methods section. There is no statistically significant difference between the values in UAS-GFP-expressing discs ( $n=23, P>0.05$ both for anti-Caspase-3 and anti-Hid). A statistically significant reduction in the values of the posterior compartment is observed when UAS-dronc-RNA-i ( $n=19, P<0.0001$ for both markers), UAS-dp53-RNA-i ( $n=20, P<0.01$ for anti-Caspase-3 and $P<0.001$ for anti-Hid) or UAS-puc ( $n=27, P<0.01$ for anti-Caspase-3 and $P<0.001$ for anti-Hid) are crossed to en-Gal4 UAS-GFP flies. ${ }^{\star} P<0.01,{ }^{\star \star} P<0.001,{ }^{* \star \star} P<0.0001$

This rules out the possibility that interference with the loop components affects the synthesis of the exogenous Hid protein. Together, these experiments clearly establish that the $d p 53 / \mathrm{JNK}$ feedback loop amplifies the apoptotic program by inducing additional proapoptotic genes.

We also analyzed the involvement of the feedback loop in the apoptotic response to physiological stress. Previous work $^{22}$ has shown that irradiation induces high levels of JNK activity in the wing disc, as indicated by the activity of the target gene puc. We reasoned that at least part of the JNK activity should be because of its activation downstream dronc, and then we designed experiments to measure and compare JNK activity levels after irradiation in the presence and absence of dronc activity. We have tested JNK activity $4 \mathrm{~h}$ after irradiation in two different genotypes. (1) Directly in dronc $^{-}$, puc ${ }^{E 69}-\mathrm{lacZ} /+$ mutant discs. This is possible because the null trans-heterozygous combination dronc ${ }^{129}$ / dronc $^{124}$ allows normal larval viability up to the pupal stage. ${ }^{29}$ (2) In discs of genotype en $>$ diap1; puc ${ }^{E 69}$-lacZ/+, in which the overexpression of diap1 in the posterior compartment results in partial suppression of dronc function. ${ }^{30}$ In this experiment, the anterior compartments serve as controls because dronc function is unaffected.
The results are illustrated in Figure 7. In dronc ${ }^{-}$discs, puc expression after irradiation (Figure $7 b$ and $d$ ) is much lower than in the dronc $^{+}$controls (Figure 7a). Similarly, in the en $>$ diap1 discs the expression levels of puc in the posterior compartment, where dronc activity is compromised, is much lower than in the control anterior compartment (Figure 7c). Taken together, these experiments establish that much of JNK function in stress-induced apoptosis derives from its activation downstream dronc, thus illustrating the role of the feedback loop in a physiological context. In addition, they bear on the mechanism by which the JNK pathway is activated after stress. It has been shown ${ }^{31}$ that the physiological response of JNK to stress depends on dTraf1 function. Our results therefore suggest an interaction between Dronc and dTraf1 in order to activate JNK.

\section{Discussion}

In Drosophila, like in other organisms, apoptosis may be developmentally regulated as part of the normal morphogenetic process ${ }^{32,33}$ or may function as a safeguard mechanism to remove damaged or malignant cells. ${ }^{12,34}$ The latter mechanism is activated after stress events that cause 

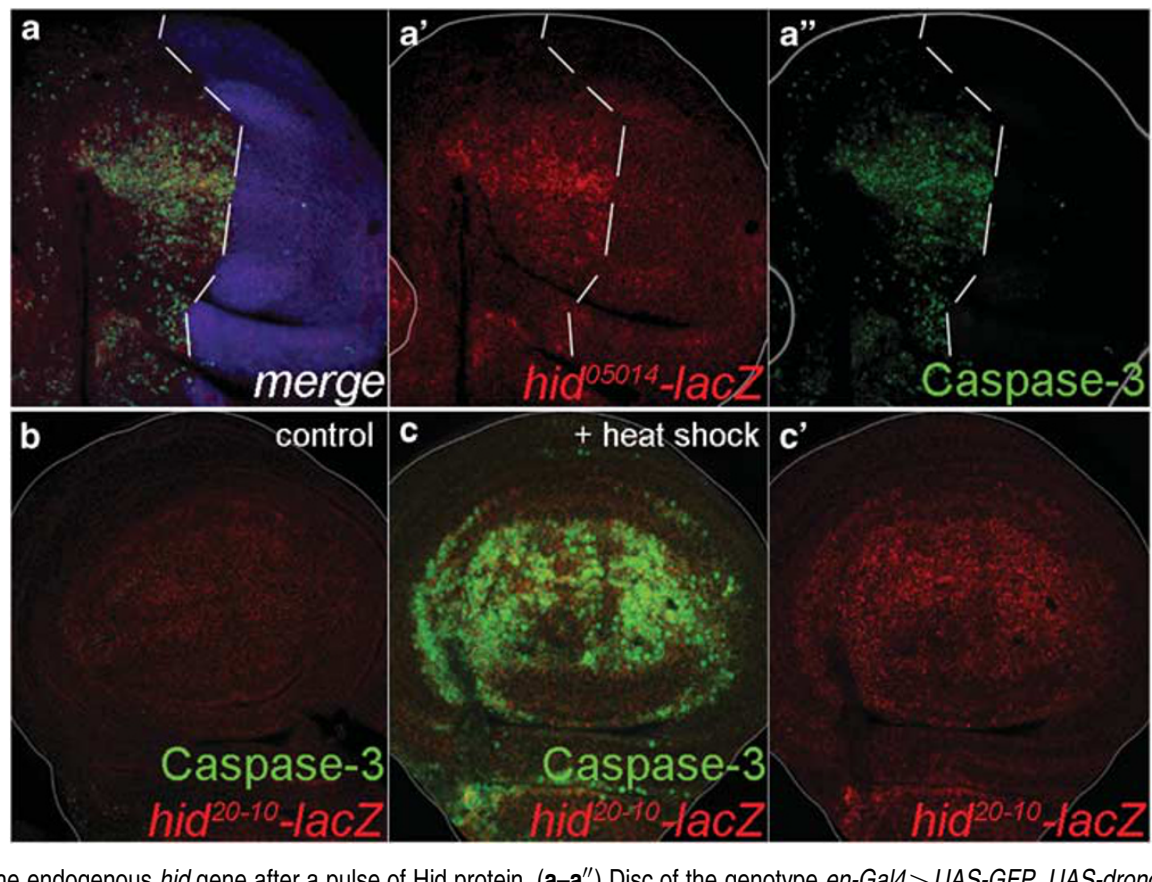

Figure 6 Activation of the endogenous hid gene after a pulse of Hid protein. (a-a $\left.\mathbf{a}^{\prime \prime}\right)$ Disc of the genotype en-Gal4> UAS-GFP, UAS-dronc RNA-i, hid ${ }^{\text {D5014-lacZ; }}$ hs-hid fixed $4 \mathrm{~h}$ after the end of the heat shock, labelled for $\beta$-gal (red) and caspase (green). The posterior compartment is labelled in blue. The panel (a') shows lacZ activity in the anterior compartment, which is largely coextensive with caspase activity, shown in $\left(\mathbf{a}^{\prime \prime}\right)$. (b) Non-heat shocked disc of genotype $h s$-hid/hid ${ }^{20-10}$-lacZ showing background levels of lacZ expression. (c and $\mathbf{c}^{\prime}$ ) Disc of the same genotype fixed $4 \mathrm{~h}$ after heat shock. lacZ expression is induced and is coextensive with high levels of caspase activity

extensive cell damage. Our results bear mainly on stressinduced apoptosis. Currently, the apoptotic program in Drosophila is portrayed as a lineal cascade of events, like that in Figure 1a. Apoptotic stimuli are channeled by upstream activators like $d p 53$ and/or JNK, which activate one or more of the proapoptotic genes hid, rpr or grim. These in turn initiate the cell death process by suppressing diap1 and allowing caspase activation.

Our results provide a new picture of the mode of action of dp53and JNK in apoptosis; not only they function as upstream activators of the proapoptotic genes in response to stress like DNA damage, but they also act downstream dronc to amplify the apoptotic program by secondary activation of additional proapoptotic genes.

We suggest a model of stress-induced apoptosis (Figure 8) in which most of the cell death is caused by the secondary activation of $d p 53$ and JNK downstream the apical caspase dronc. Because $d p 53$ and JNK are able to induce hid and rpr, it results in the establishment of a loop that amplifies the original apoptotic stimulus. The fact that $d p 53$ and JNK activate each other (Figure 3) also contributes to the loop. Our results also indicate that the loop operates in the physiological response to stress events (Figures 7a-d).

This amplification appears to be an essential and necessary component of the apoptotic program of Drosophila because its suppression or reduction causes a dramatic decrease of cell death. This is clearly shown by the heat shock experiments (Figures 4 and 5 and Supplementary Figure S3) in which we force the initiation of the apoptotic program by providing the proapoptotic proteins Hid or Rpr; the amount of cell death after the stimulus depends critically on the existence of the amplification loop.
The loop also provides a mechanism of mutual activation of proapoptotic genes; as indicated in Figures $2 \mathrm{e}$ and $\mathrm{h}$, the activation of hid gives rise to rpr function, and conversely rpr function induces hid (Supplementary Figure S1). This mutual activation may be an important part of the completion of the program because it has been shown in a recent report ${ }^{30}$ that the Rpr and Hid proteins interact physically and that their association is critical for their cell killing activity. The loopmediated mutual activation of hid and rpr may be instrumental to provide the right amounts of Rpr and Hid proteins needed for the interaction.

We also find that $d p 53$ and JNK are able to activate each other even in discs in which apoptosis is suppressed. The mechanistic aspects of these interactions are not clear; it has been shown that the ligand Eiger is a target of Dp53, ${ }^{35}$ suggesting it may mediate JNK activation by $d p 53$. How $d p 53$ is transcriptionally activated by JNK is not known, although it might contribute to the stabilization of the Dp53 protein (see below).

The amplification loop may be a mechanism to add robustness to the apoptotic program; it is possible to imagine a situation in which a weak apoptotic stimulus may induce the proapoptotic genes at low levels. Depending on a threshold of dronc function, it may result in the establishment of the amplification loop and the death of the cell, or else the loop is not established and the cell survives.

Although we believe our experiments clearly demonstrate the existence of an amplification loop in stress-induced apoptosis, it is not clear whether it also occurs in developmentally regulated apoptosis. Nevertheless, there are indications that the loop functions in some developmental contexts. In the leg disc, it has been shown that the correct formation of 

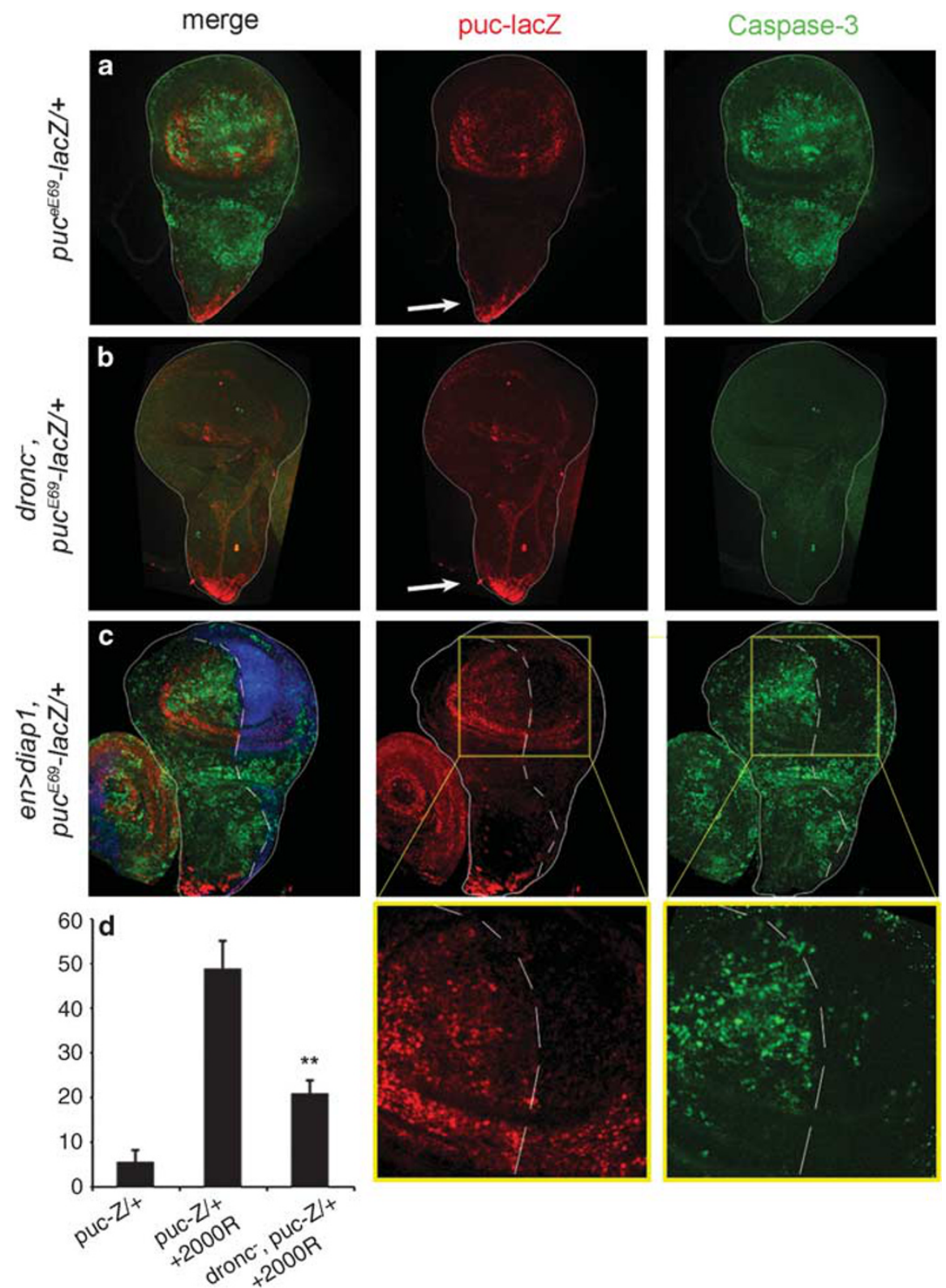

Figure 7 Activation of the JNK pathway after physiological stress. (a) Control puc ${ }^{E 69}$-lacZ/ + disc showing high levels of puc expression and caspase activity in the wing pouch $4 \mathrm{~h}$ after an irradiation of 2000R. The expression of puc in the proximal region (arrow) is normally present and corresponds to the midline cells. (b) Disc of genotype drond $^{124}$, puc ${ }^{E 69}-$ lacZ/dronc ${ }^{129}$ showing very low expression of puc and caspase activity after the same dose of irradiation as in (a). Note (arrow) the normal puc expression in the midline cells. (c) Disc of genotype en $>$ diap1; puc ${ }^{E 69}-$ lacZl + . The low dronc activity in the posterior compartment results in low level of puc expression and caspase activity after a 2000R radiation. (d) The left panel shows a quantification of puc levels (see Materials and Methods) in a normal nonirradiated puc ${ }^{E 69}-$ lacZ/ + disc, in an irradiated disc of the same phenotype and in an irradiated $d r o n c^{124}, p u c^{E 69}-$ lacZ/dronc $c^{129}$ disc. The middle and right panels show amplification of the inset in the corresponding photographs in (c). ${ }^{* *} P<0.001$

the leg articulations requires local apoptosis in the intersegmental regions, ${ }^{33}$ where $r p r$ and the JNK pathway are coexpressed. We have observed that this local JNK activity is suppressed in dronc mutant discs (Supplementary Figure S6), indicating it is downstream dronc, in good concordance with the loop hypothesis.

There are also some indications of the existence of apoptosis amplification loops in vertebrates. For example, in mammalian cells activated caspases feedback to amplify cytochrome $c$ release and hence augment caspase-9 activity, ${ }^{36}$ a process that could be mediated by $p 53 .{ }^{37}$ It has also been proposed that in mammalian cells phosphorylation of P53 at Ser6 by JNK contributes to its stability and promotes its proapoptotic role. ${ }^{38}$ These observations suggest functional interactions between caspases, p53 and JNK, similar to those we describe for Drosophila that may result in the establishment of an apoptotic loop.

The antitumor properties of $p 53$ and JNK pathway derive in part from their ability to induce apoptosis. Our results indicate that in Drosophila apoptosis, $d p 53$ and the JNK pathway are 


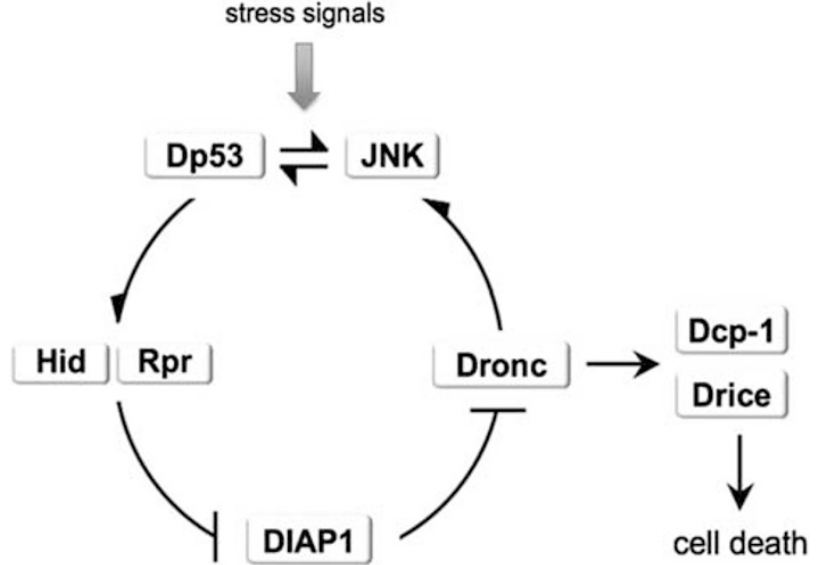

Figure 8 Amplification loop model of stress-induced apoptosis. We envisage stress-induced apoptosis as follows: an original stress event (irradiation, heat shock) induces $d p 53$ function, which activates hid and rpr transcription and also the JNK pathway. The JNK pathway is also able to amplify dp53 transcription (and probably to induce rpr and hid as well). The activation of dronc because of Hid/Rpr function gives rise, on one hand, to the activation of effector caspases and, on the other, to an increase of both Dp53 and JNK levels, thus completing the amplification loop. This feedback is necessary for the completion of the apoptotic program, because in its absence there is a dramatic reduction in the amount of cell death. The model implies that the cell killing function of Dronc requires amplification of its own levels. This is achieved by its ability to induce $d p 53 / J N K$ function. The model incorporates the functional interactions among the various factors involved, but it does not contemplate their interactions at the molecular level. Some of the molecular interactions between Hid, Rpr, Diap1, Dronc and Drice are well known, ${ }^{6,7}$ but the mechanisms of activation of $d p 53$ and JNK by Dronc and the specific molecular interactions between $d p 53$ and JNK have not yet been described

activated downstream of caspase-9/Dronc and that this activation is critical for the completion of the process. An intriguing possibility is that in humans much of the proapoptotic and hence tumor-suppressive function of P53 and JNK is exerted downstream caspase-9. It might have implications in pharmacological aspects of cancer therapies, as altering caspase activity could be a vehicle to modify P53/JNK signalling. In this sense, it may be of interest to examine P53/JNK activity levels in human tumors that bear mutations in caspases.

\section{Materials and Methods}

Drosophila stocks. $h$ h-Gal4 and en-Gal4 (T Tabata, IMBC, Tokyo, Japan) direct expression to the posterior compartment, and the sal ${ }^{E P V}$-Gal4 in part of the wing pouch (gift of JF de Celis, CBMSO, Madrid, Spain). UAS-dronc-RNA-i (no. 23035) and UAS-dp53-RNA-i (no. 10692) were obtained from Vienna Drosophila RNA-i Center (Vienna, Austria). UAS-puc ${ }^{2 A}, U A S-p 35$, UAS-dp53.EX 2, UAS-dp53 ${ }^{D N}, U A S-h e p^{A C T}$, UAS-hid and UAS-rpr are described in Flybase.

The puc ${ }^{E 69}$-lacZ line ${ }^{27}$ was used as a reporter of JNK pathway activity. We obtained from T Adachi-Yamada (Kobe University, Kobe, Japan) the hid ${ }^{05014}-$-lacZ, a P-element insertion in hid coding region, ${ }^{28}$ because it has been used previously as a reporter of hid activity. ${ }^{39}$ As this line has a nonapoptotic expression in some anterior cells close to the dorsal-ventral boundary in late third-instar larval stage, we used the hid ${ }^{20-10}-l a c Z,{ }^{11}$ which lacks nonspecific expression (see Figure $6 \mathrm{~b}$ ), to confirm our results. We obtained this line from HD Ryoo (NYU, New York, NY, USA). $d r o n c^{124}$ and $d r o n c^{129}$ are considered null alleles, ${ }^{29}$ and were contributed by $\mathrm{A}$ Bergmann (MD Anderson Center, Houston, TX, USA). We recombined puc ${ }^{E 69}$ with $d^{2} c^{124}$, and $h s-h i d^{3}$ and $h s-r p r^{37 B}$ are gifts of H Steller (HHMI, New York, NY, USA) and have been previously described. ${ }^{28,40}$
Quantitative analysis. In each analysis, all the discs were treated in the same condition (see Inmunostaining section) and images were processed using the Image J software (NIH, Bethesda, MD, USA) as follows. Each image analyzed was a representative section of the wing disc. A high-intensity threshold that corresponded to the labelling with the antibody was adjusted for each image. Then, we generated a region of interest (ROI) that encompassed the compartment under study and calculated the percentage of the $\mathrm{ROI}$ covered by the staining (pixels that are above of the threshold level) using the Area Fraction option in Set Measurements. The number of discs analyzed in each experiment is given in the figure legends. The $P$-values were calculated using two-tailed Student's $t$-test in Microsoft Excel.

Inmunostaining and in situ hybridization. Inmunostaining was performed largely as described previously, ${ }^{22}$ with slight modifications. Wing imaginal discs from wandering third-instar larvae were dissected in ice-cold PBS and fixed in $4 \%$ paraformaldehyde, $0.1 \%$ Triton and $0.1 \%$ DOC for $30 \mathrm{~min}$ at room temperature. Discs were subsequently blocked in blocking buffer (PBS, $0.3 \%$ Triton and $1 \% \mathrm{BSA}$ ) and incubated overnight at $4{ }^{\circ} \mathrm{C}$ with the primary antibody diluted in PBT (PBS, $0.3 \%$ Triton). Washes were performed in PBT and incubation with the secondary antibody was carried for $2 \mathrm{~h}$ at room temperature. Following further washing, discs were mounted in Vectashield (Vector Laboratories Inc., Burlingame, CA, USA). All images were acquired with a Leica (Solms, Germany) DB5500 B confocal microscope except double antibody staining and in situ hybridization images, which were acquired with a LSM510 Meta (Zeiss, Thornwood, NY, USA) confocal microscope.

Primary antibodies were used in the following dilutions: mouse anti- $\beta$-gal (Promega, Madison, WI, USA) 1: 1000; guinea pig anti-Hid (a gift from Hyung Don Ryoo, NYMC, New York, NY, USA), 1:200; rabbit anti-cleaved Caspase-3 (Cell Signaling Technology, Danvers, MA, USA), 1:50; mouse anti-pJNK (Cell Signaling), 1:100; rabbit anti-GFP (Molecular Probes, Inc., Eugene, OR, USA), 1:300; and anti-Digoxigenin-AP (Roche, Basel, Switzerland), 1:4000. Molecular Probes Alexa secondary antibodies were used in a 1:200 dilution.

For double antibody staining and in situ hybridization, the in situ hybridization was performed as described elsewhere with the following modification: in situ hybridization was followed by incubation with the primary antibody (e.g., anti-Caspase-3 in Figure 6b), anti-GFP and anti-DIG-AP overnight at $4^{\circ} \mathrm{C}$ After washing the discs were incubated with secondary antibodies, always including in the mix one that recognized the species of the anti-GFP, and also anti-DIG (a second round of incubation with the primary antibody to amplify the signal), for $2 \mathrm{~h}$ at room temperature. The hybridization pattern was revealed using Roche Alkaline Phosphatase reagents NBT (no. 11383213 001) and BCIP (no. 11383221001 ) in $1 \mathrm{M}$ Tris- $\mathrm{HCl}$, $\mathrm{pH} 9.5$.

RNA probe for the $d p 53$ transcript was obtained using the BDGP EST GH11591 clone as a template. rpr probe is a gift of I Lohmann (MPI for Developmental Biology, Tübingen, Germany).

\section{Conflict of Interest}

The authors declare no conflict of interest.

Acknowledgements. We thank Ernesto Sanchez-Herrero for fruitful discussions, Francisco A Martín and Hermann Steller for critical reading of the manuscript and Ana Guarner for help with leg preparations. We also thank Andreas Bergmann for dronc mutants, Hermann Steller for the heat shock stocks, Jose F de Celis for the sal ${ }^{E P v}$-Gal4 line, Don Ryoo for the anti-Hid antibody, Ingrid Lohmann for the rpr probe and the Confocal Microscopy Service at the CBMSO for help with acquisition and processing of images. We also thank Angelica Cantarero and Rosa Gonzalez for general help and the members of the lab for discussions. The experimental work has been funded by grants from the Ministerio de Ciencia e Innovación (Consolider CSD 20700008-B and BFU 2008 03196/BMC) and by an institutional grant from the Fundación Ramón Areces. ES is supported by a FPU scholarship (AP2005-4875).

1. Jacobson MD, Weil M, Raff MC. Programmed cell death in animal development. Cell 1997; 88: 347-354.

2. Meier $P$, Finch A, Evan G. Apoptosis in development. Nature 2000; 407: 796-801.

3. Thornberry NA, Lazebnik Y. Caspases: enemies within. Science 1998; 281: 1312-1316.

4. Reed JC, Doctor KS, Godzik A. The domains of apoptosis: a genomics perspective. Sci STKE 2004; 2004: re9.

5. Shiozaki EN, Shi Y. Caspases, IAPs and Smac/DIABLO: mechanisms from structural biology. Trends Biochem Sci 2004; 29: 486-494. 
6. Hay BA, Guo M. Caspase-dependent cell death in Drosophila. Annu Rev Cell Dev Biol 2006; 22: 623-650.

7. Wilson R, Goyal L, Ditzel M, Zachariou A, Baker DA, Agapite J et al. The DIAP1 RING finger mediates ubiquitination of Dronc and is indispensable for regulating apoptosis. Nat Cell Biol 2002; 4: 445-450.

8. Steller H. Drosophila p53: meeting the Grim Reaper. Nat Cell Biol 2000; 2: E100-E102.

9. Riley $\mathrm{T}$, Sontag E, Chen $\mathrm{P}$, Levine A. Transcriptional control of human p53-regulated genes. Nat Rev Mol Cell Biol 2008; 9: 402-412.

10. Zilfou JT, Lowe SW. Tumor suppressive functions of p53. Cold Spring Harb Perspect Biol 2009; 1: a001883.

11. Fan Y, Lee TV, Xu D, Chen Z, Lamblin AF, Steller H et al. Dual roles of Drosophila p53 in cell death and cell differentiation. Cell Death Differ 2010; 17: 912-921.

12. Brodsky MH, Nordstrom W, Tsang G, Kwan E, Rubin GM, Abrams JM. Drosophila p53 binds a damage response element at the reaper locus. Cell 2000; 101: 103-113.

13. Kanda H, Miura M. Regulatory roles of JNK in programmed cell death. J Biochem 2004; 136: $1-6$

14. Adachi-Yamada T, O'Connor MB. Morphogenetic apoptosis: a mechanism for correcting discontinuities in morphogen gradients. Dev Biol 2002; 251: 74-90.

15. Igaki T. Correcting developmental errors by apoptosis: lessons from Drosophila JNK signaling. Apoptosis 2009; 14: 1021-1028.

16. Hay BA, Wolff $T$, Rubin GM. Expression of baculovirus P35 prevents cell death in Drosophila. Development 1994; 120: 2121-2129.

17. Martin FA, Perez-Garijo A, Morata G. Apoptosis in Drosophila: compensatory proliferation and undead cells. Int J Dev Biol 2009; 53: 1341-1347.

18. Perez-Garijo A, Martin FA, Morata G. Caspase inhibition during apoptosis causes abnormal signalling and developmental aberrations in Drosophila. Development2004; 131 : $5591-5598$

19. Wells BS, Yoshida E, Johnston LA. Compensatory proliferation in Drosophila imaginal discs requires Dronc-dependent p53 activity. Curr Biol 2006; 16: 1606-1615.

20. McEwen DG, Peifer M. Puckered, a Drosophila MAPK phosphatase, ensures cell viability by antagonizing JNK-induced apoptosis. Development 2005; 132: 3935-3946.

21. Ryoo HD, Gorenc T, Steller H. Apoptotic cells can induce compensatory cell proliferation through the JNK and the Wingless signaling pathways. Dev Cell 2004; 7: 491-501.

22. Perez-Garijo A, Shlevkov E, Morata G. The role of Dpp and $\mathrm{Wg}$ in compensatory proliferation and in the formation of hyperplastic overgrowths caused by apoptotic cells in the Drosophila wing disc. Development 2009; 136: 1169-1177.

23. Brand AH, Perrimon N. Targeted gene expression as a means of altering cell fates and generating dominant phenotypes. Development 1993; 118: 401-415.

24. Cruz C, Glavic A, Casado M, de Celis JF. A gain-of-function screen identifying genes required for growth and pattern formation of the Drosophila melanogaster wing. Genetics 2009; 183: 1005-1026.
25. Luo X, Puig O, Hyun J, Bohmann D, Jasper H. Foxo and Fos regulate the decision between cell death and survival in response to UV irradiation. EMBO J 2007; 26: 380-390.

26. Adachi-Yamada T, Fujimura-Kamada K, Nishida Y, Matsumoto K. Distortion of proximodistal information causes JNK-dependent apoptosis in Drosophila wing. Nature 1999; 400: 166-169.

27. Martín-Blanco E, Gampel A, Ring J, Virdee K, Kirov N, Tolkovsky AM et al, puckered encodes a phosphatase that mediates a feedback loop regulating JNK activity during dorsal closure in Drosophila. Genes Dev 1998; 12: 557-570.

28. Grether ME, Abrams JM, Agapite J, White K, Steller H. The head involution defective gene of Drosophila melanogaster functions in programmed cell death. Genes Dev 1995; 9 1694-1708.

29. Xu D, Li Y, Arcaro M, Lackey M, Bergmann A. The CARD-carrying caspase Dronc is essential for most, but not all, developmental cell death in Drosophila. Development 2005; 132: 2125-2134.

30. Shapiro PJ, Hsu HH, Jung H, Robbins ES, Ryoo HD. Regulation of the Drosophila apoptosome through feedback inhibition. Nat Cell Biol 2008; 10: 1440-1446.

31. Kuranaga $\mathrm{E}$, Kanuka $\mathrm{H}$, Igaki T, Sawamoto $\mathrm{K}$, Ichijo $\mathrm{H}$, Okano $\mathrm{H}$ et al. Reaper-mediated inhibition of DIAP1-induced DTRAF1 degradation results in activation of JNK in Drosophila. Nat Cell Biol 2002; 4: 705-710.

32. Lohmann I, McGinnis N, Bodmer M, McGinnis W. The Drosophila Hox gene deformed sculpts head morphology via direct regulation of the apoptosis activator reaper. Cell 2002; 110: $457-466$.

33. Manjon C, Sanchez-Herrero E, Suzanne M. Sharp boundaries of Dpp signalling trigger local cell death required for Drosophila leg morphogenesis. Nat Cell Biol 2007; 9: 57-63.

34. Menendez J, Perez-Garijo A, Calleja M, Morata G. A tumor-suppressing mechanism in Drosophila involving cell competition and the Hippo pathway. Proc Natl Acad Sci USA 2010; 107: 14651-14656

35. Brodsky MH, Weinert BT, Tsang G, Rong YS, McGinnis NM, Golic KG et al. Drosophila melanogaster MNK/Chk2 and p53 regulate multiple DNA repair and apoptotic pathways following DNA damage. Mol Cell Biol 2004; 24: 1219-1231.

36. Lakhani SA, Masud A, Kuida K, Porter Jr GA, Booth CJ, Mehal WZ et al. Caspases 3 and 7 : key mediators of mitochondrial events of apoptosis. Science 2006; 311: 847-851.

37. Sayan BS, Sayan AE, Knight RA, Melino G, Cohen GM. p53 is cleaved by caspases generating fragments localizing to mitochondria. J Biol Chem 2006; 281: 13566-13573.

38. Fuchs SY, Adler V, Pincus MR, Ronai Z. MEKK1/JNK signaling stabilizes and activates 553. Proc Natl Acad Sci USA 1998; 95: 10541-10546.

39. Adachi-Yamada T, Harumoto T, Sakurai K, Ueda R, Saigo K, O'Connor MB et al. Wing-toLeg homeosis by spineless causes apoptosis regulated by Fish-lips, a novel leucinerich repeat transmembrane protein. Mol Cell Biol 2005; 25: 3140-3150.

40. White K, Tahaoglu E, Steller H. Cell killing by the Drosophila gene reaper. Science 1996 271: 805-807.

\section{Supplementary Information accompanies the paper on Cell Death and Differentiation website (http://www.nature.com/cdd)}

